\title{
Preparation of Silver Nanocolloids Using Silver Alkylcarbamate Complex in Organic Medium with PVP Stabilizer
}

\author{
Hyung-Seok Park, Heon-Su Park, and Myoung-Seon Gong* \\ Department of Nanobiomedical Science and WCU Research Center of Nanobiomedical Science, Chungnam 330-714, Korea \\ *E-mail:msgong@dankook.ac.kr \\ Received June 21, 2010, Accepted July 26, 2010
}

\begin{abstract}
We present a method for chemically reducing silver alkylcarbamate complex with hydrazine to synthesize silver nanocolloids in an organic solvent using polyvinylpyrrolidone (PVP) as the stabilizer. To determine the optimal conditions for preparing stable silver colloids of controlled size and shape, the silver 2-ethylhexylcarbamate (AgEHCB) complex, PVP, hydrazine, and 2-propanol solvent concentrations in the reaction mixture were varied. The initial colloid has a mean particle diameter of $5-80 \mathrm{~nm}$, and it exhibits an absorption band with various shapes in the UV region with a maximum near $420 \mathrm{~nm}$. UV-vis spectroscopy, TEM, and X-ray diffraction techniques were used to investigate the formation and growth process of the metallic silver nanocolloids.
\end{abstract}

Key Words: Silver nanocolloid, Polyvinylpyrrolidone, Silver alkylcarbamate, Organic solvent, Hydrazine

\section{Introduction}

Various chemical methods have been used to synthesize metallic nanoparticle dispersions, the most common of these approaches uses an excess amount of a reducing agent, such as sodium citrate ${ }^{1}$ or sodium borohydride. ${ }^{2,3}$ Other simple methods involve reducing metallic salts by the solvent used to prepare colloidal dispersions. ${ }^{4-9}$ Hydrogen reduction techniques represent some of the simplest ways to obtain colloidal dispersions because they require a minimum number of components, thus avoiding the need for an undesirable excess of the reducing agent and the formation of reaction byproducts. ${ }^{10}$ However, hydrazine reduction method offers the advantages of being able to produce the essentially also clean silver particles, which contains no inorganic ions except amine, carbon dioxide and nitrogen, even at room temperature.

Several silver salt precursors have been used for the synthesis of silver nanoparticles (NPs). $\mathrm{AgNO}_{3}{ }^{1,11-12}$ is the most common source of silver ions; however, $\mathrm{Ag}_{2} \mathrm{SO}_{4}$, ${ }^{1}$ silver 2-ethylhexanoate, ${ }^{6}$ silver oxide, ${ }^{13}$ and silver perchlorate ${ }^{14}$ also have been used. Generally, the actual size of the obtained NPs varies from system to system because the stabilizer, reducing agent and nature of the metal can be varied and because parameters such as solvent, concentration, temperature, and reduction time are different.

Silver organic salts have been used to produce silver NPs and conductive silver tracks. In these processe silver carboxylate $^{15-24}$ and silver alkylcarbamate complexes are reduced to silver metal by heating. ${ }^{25-31}$ However, a limited number of reports have described the deposition of silver onto substrates via the one-pot reduction of silver alkylcarbamate complex in solution using hydrazine as a reducing agent. ${ }^{32,33}$ However, no other reports have described the formation of silver nanocolloids via the one-pot reduction of silver alkylcarbamate complex in organic solution using hydrazine as the reducing agent. This method offers the advantage of producing the essentially clean silver particles containing no inorganic ions except amine and carbon dioxide, even at room temperature.

Numerous preparation methods for these colloidal metal dispersions use polymers during reduction of the metal precursors. Thus, the polymer can profoundly influence the particle features of the resulting metal colloids and their long-term colloidal stability. $^{34-39}$

This work describes the preparation of silver NPs by simple reduction of Ag-EHCB complex with hydrazine in 2-propanol solution. Polyvinylpyrrolidone was investigated for its ability to stabilize colloidal silver nanoparticles and the subsequent effects on nanoparticle features. Conditions of the Ag-EHCB reduction via hydrazine were systematically varied to investigate their effects on the size and morphological characteristics of the silver NPs. Finally, the optimal experimental conditions, such as amount of solvent, concentration of Ag-EHCB, and type of stabilizer used for the preparation of the silver NPs, and the reduction mechanism were also determined.

\section{Experimental}

Chemicals and instruments. The silver 2-ethylhexylcarbamate complex (Ag-EHACB, $20 \mathrm{wt} \%$ ) solution used throughout this work as the precursor was purchased from Inktec Co., Ltd. A predetermined quantity of PVP (MW 40,000, Aldrich Chem. Co.) was added to the solution of Ag-EHACB in 2-propanol. Using a reducing agent solution prepared from hydrazine monohydrate (Aldrich Chem. Co.) and PVP, we tested the effect of reducing agent quantity by varying the hydrazine concentration from $2 \times 10^{-2}$ to $7 \times 10^{-1} \mathrm{~mol} / \mathrm{L}$. Formation of silver colloids can be achieved in less than 20 min over the entire tested range of hydrazine concentrations. Variation of the size distribution over time was determined. The stability of these colloids was tested at several conditions. XRD and TEM also were applied to obtain information on the structure and morphology of these colloids.

TEM observation was carried out using a JEOL electron microscope (JEM-2000EXII). Prior to measurement, samples 
were prepared by dropping a small amount of silver colloid solution on a carbon-coated copper grid. After the solvent was evaporated, the particles were sized. The silver nanocolloid solutions were diluted in 2-propanol and placed on the cell of a quartz cuvette with a path length of $1 \mathrm{~cm}$; the incident light beam was oriented perpendicular to the radial direction of the samples. The UV-vis spectra were obtained using a Shimadzu UV-1601PC spectrometer from 200 to $800 \mathrm{~nm}$, with a resolution of $1 \mathrm{~nm}$ and background correction, using 2-propanol as the solvent. All the UV-vis spectra were obtained from the original colloid solution diluted with an appropriate amount of 2-propanol. The XRD patterns of the Ag NPs were measured using a Shimadzu XD-D1 X-ray diffractometer with $\mathrm{Cu} \mathrm{K \alpha}$ radiation $(\lambda=1.54056 \AA)$ at a scanning rate of 2 degrees per second in the $2 \theta$ range from $30^{\circ}$ to $90^{\circ}$. The sample for XRD analysis was supported on a glass substrate.

Preparation of silver nanocolloid solution. The reducing agent, hydrazine monohydrate (1.0 g), and stabilizer, PVP $(10 \mathrm{~g})$, were dissolved in 2-propanol $(200 \mathrm{~mL})$. The precursor, Ag-EHCB complex (1.0 g), and PVP (10 g) also were dissolved in 2-propanol $(200 \mathrm{~mL})$ in another container. The solution of hydrazine and PVP was added dropwise to the solution of PVP and Ag-EHCB complex for $20 \mathrm{~min}$ at $25{ }^{\circ} \mathrm{C}$ to yield the silver nanocolloid solution. Additional silver nanocolloid solutions with varying contents of PVP, Ag-EHCB complex, and solvent were prepared by procedures similar to those described above.

\section{Results and Discussion}

Preparation of silver colloids. The Ag-EHCB complex and hydrazine were used as the silver precursor and reducing agent, respectively, for preparation of the silver nanocolloid. To the best of our knowledge, this is the first description of the preparation of a silver nanocolloid solution using a silver alkylcarbamate complex solution with hydrazine.

Several stabilizers were tested, which permitted us to determine that the nature and concentration of the stabilizers are crucial parameters for both the yield of the colloidal solution and the stability of the particles. PVP was selected as a stabilizer for the dispersion of the silver NPs in 2-propanol solution. When the reducing agent solution was poured into the Ag-EHCB complex solution, the solution changed color to reddish black. At this time, the precursor was rapidly reduced to produce silver NPs.

To determine the optimal conditions for preparing stable silver nanocolloids of varying size, shape, and particle size distributions, a large number of experiments were conducted while varying the concentrations of hydrazine, Ag-EHCB complex, $\mathrm{PVP}$, and 2-propanol in the reaction mixture, as shown in Table 1.

Effect of hydrazine concentration. The completeness of the Ag-EHCB complex reduction was followed by UV-vis spectroscopy, specifically by monitoring the appearance of the absorption bands of the silver surface plasmon near $420 \mathrm{~nm}$. The

Table 1. Conditions for the preparation of silver particles using silver alkylcarbamate complex solution by reduction with hydrazine

\begin{tabular}{|c|c|c|c|c|c|c|c|}
\hline Exp. No. & $\begin{array}{l}{[\mathrm{PVP}]^{a} \times 10^{3}} \\
(\mathrm{~mol} / \mathrm{L})\end{array}$ & $\underset{(\mathrm{mol} / \mathrm{L})}{\left[\begin{array}{l}\text { hydrazine } \\
b\end{array}\right.} \times 10$ & $\underset{(\mathrm{mol} / \mathrm{L})}{[\mathrm{Ag}-\mathrm{EHCB}]^{c}} \times 10^{3}$ & $\begin{array}{l}\mathrm{IPA}^{d} \\
(\mathrm{~L})\end{array}$ & $\begin{array}{l}\text { Time } \\
(\min )\end{array}$ & {$[$ Hydrazine $] /[\mathrm{Ag}]^{e}$} & {$[\mathrm{PVP}] /[\mathrm{Ag}]^{\dagger}$} \\
\hline 1 & \multirow{12}{*}{9.0} & 0.2 & \multirow{8}{*}{4.64} & \multirow{24}{*}{0.40} & \multirow{16}{*}{20} & 2.16 & \multirow{8}{*}{1.94} \\
\hline 2 & & 0.4 & & & & 4.32 & \\
\hline 3 & & 0.8 & & & & 8.64 & \\
\hline 4 & & 1.0 & & & & 10.78 & \\
\hline 5 & & 1.5 & & & & 16.17 & \\
\hline 6 & & 2.0 & & & & 21.56 & \\
\hline 7 & & 3.0 & & & & 32.34 & \\
\hline 8 & & 5.0 & & & & 53.90 & \\
\hline 9 & & 0.2 & 0.93 & & & \multirow{8}{*}{10.78} & 9.70 \\
\hline 10 & & 0.5 & 2.32 & & & & 3.88 \\
\hline 11 & & 2.0 & 9.28 & & & & 0.97 \\
\hline 12 & & 5.0 & 23.20 & & & & 0.39 \\
\hline 13 & 4.5 & \multirow{4}{*}{1.0} & \multirow{12}{*}{4.64} & & & & 0.97 \\
\hline 14 & 13.5 & & & & & & 2.91 \\
\hline 15 & 18.0 & & & & & & 3.88 \\
\hline 16 & 45.0 & & & & & & 9.70 \\
\hline 17 & \multirow{8}{*}{9.0} & 0.2 & & & 5 & 2.16 & \multirow{8}{*}{1.94} \\
\hline 18 & & 0.4 & & & 10 & 4.32 & \\
\hline 19 & & 0.6 & & & 12 & 6.48 & \\
\hline 20 & & 0.8 & & & 15 & 8.64 & \\
\hline 21 & & 2.0 & & & 30 & 21.56 & \\
\hline 22 & & 2.0 & & & 40 & 21.56 & \\
\hline 23 & & 4.0 & & & 60 & 43.12 & \\
\hline 24 & & 6.0 & & & 80 & 64.68 & \\
\hline
\end{tabular}

${ }^{a}$ Concentration of $20.0 \mathrm{~g}$ of PVP in 2-propanol solution $(400 \mathrm{~mL})$ was equivalent to $9.0 \times 10^{-3} \mathrm{~mol} / \mathrm{L} ;{ }^{b}$ Concentration of $1.0 \mathrm{~g}$ of hydrazine in 2 -propanol solution $(200 \mathrm{~mL})$ was equivalent to $1.0 \times 10^{-1} \mathrm{~mol} / \mathrm{L} ;{ }^{c}$ Concentration of $1.0 \mathrm{~g}$ of Ag-EHCB complex in 2-propanol solution $(400 \mathrm{~mL})$ was equivalent to $4.64 \times 10^{-3} \mathrm{~mol} / \mathrm{L}$ of silver; ${ }^{d} 2$-propanol; ${ }^{e}$ the ratio of [hydrazine] to [Ag-EHCB] in $400 \mathrm{~mL}$ of 2-propanol; ${ }^{f}$ the ratio of [PVP] to [Ag-EHCB]. 
growth rate of the silver NPs also was found to depend on the ratio of Ag-EHCB to hydrazine. Figure 1(a) shows the UV-vis spectra of the silver colloidal solutions, starting from a concentration of $9.28 \times 10^{-3} \mathrm{~mol} / \mathrm{L}$ of $\mathrm{Ag}$-EHCB in 2-propanol solution $(200 \mathrm{~mL})$. Rapid changes in UV-vis absorbance were detected as the concentration of hydrazine increased from $2.0 \times 10^{-2}$ to $7.0 \times 10^{-1} \mathrm{~mol} / \mathrm{L}$, as shown in Figure 1. It is seen that as the concentration of hydrazine increased from $2.00 \times 10^{-2} \mathrm{~mol} / \mathrm{L}$ (Exp1) to $1.5 \times 10^{-1} \mathrm{~mol} / \mathrm{L}(\operatorname{Exp} 5)$, the full width at half maximum of the absorption band increased from 85 to $102 \mathrm{~nm}$. This result suggests that the particle size distribution becomes wider and

(a)

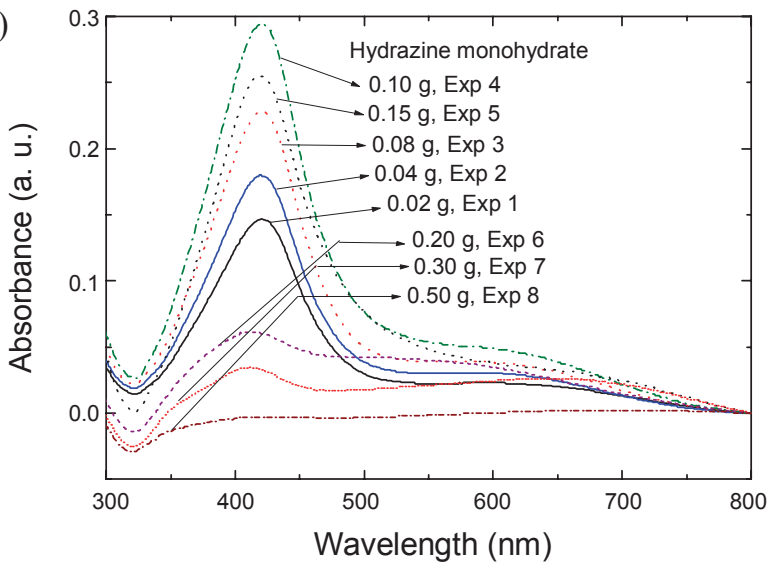

(b)

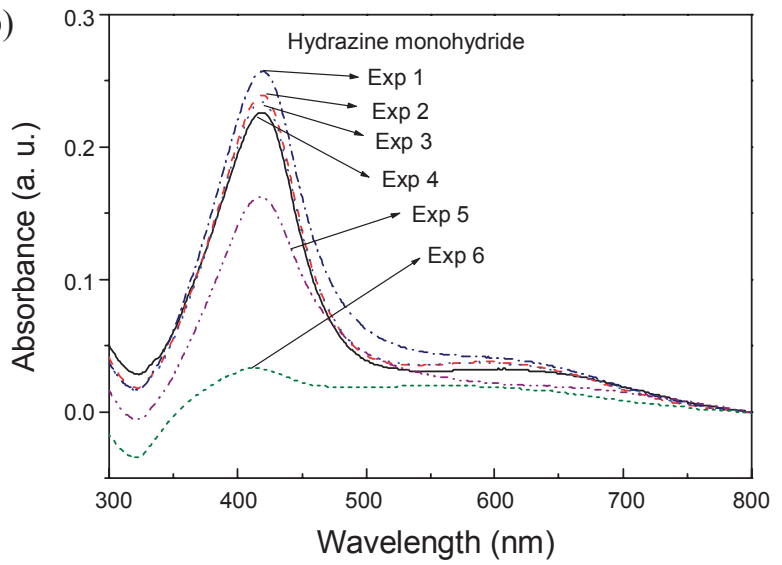

Figure 1. UV-vis absorption spectra of silver colloidal solutions obtained from the Ag-EHCB complex solution reduced with various amounts of hydrazine $\left(2 \times 10^{-2}-7 \times 10^{-1} \mathrm{~mol} / \mathrm{L}\right)$ for $20 \mathrm{~min}$ after (a) 1 day, and (b) 30 days. the colloid system changes from monodispersion to polydispersion with increasing concentrations of hydrazine. The strongest intensity and a relatively narrow particle size distribution were obtained at a hydrazine concentration of $1.0 \times 10^{-1} \mathrm{~mol} / \mathrm{L}$ for $[$ Hydrazine $] /[\mathrm{Ag}-\mathrm{EHCB}]=10.78$ and $[\mathrm{PVP}] /[\mathrm{Ag}-\mathrm{EHCB}]=$ 1.94. To monitor the stability of the final prepared silver colloid, we measured the absorption spectra of the colloid on different days, as shown in Figure 1(b). There was no obvious change in the shape, position, or asymmetry of the absorption peak during the initial 30 days. The reduction by hydrazine resulted in very stable samples, with no precipitation occurring after several months.

Figure 2 shows a typical TEM photograph of the silver nanocolloid prepared from Ag-EHCB and various concentrations of hydrazine. The smallest size (5 - $20 \mathrm{~nm}$ ) shown in Figure 2(a) is from the suspension obtained at a hydrazine concentration of $4 \times 10^{-2} \mathrm{~mol} / \mathrm{L}(\operatorname{Exp} 2)$, while the largest $(20-50 \mathrm{~nm})$ shown in Figure 2(b) corresponds to higher concentrations obtained from those in Experiment 6 ([Hydrazine $\left.]=2.0 \times 10^{-1} \mathrm{~mol} / \mathrm{L}\right)$.

As shown in Figure 2(c) most NPs were well-dispersed and polygon-shaped in Experiment 4, coinciding with the above mentioned speculation of the UV-vis absorption spectra. The particle size distribution is shown as a histogram in Figure 2(d). The Gaussian fit shows that the mean diameter of the particles is $25.8 \mathrm{~nm}$, and the fitted standard deviation is 5.0. It is obvious that the NPs in Figure 2(c) are similar in size and narrower in size distribution than those shown in Figures 2(b) and Figure 2(e) (Exp 5). Reduction with a lower concentration of hydrazine allows a relatively uniform distribution of particles to be obtained in the solution compared to that achieved at a high concentration of hydrazine. Thus, most of the silver NPs grow uniformly in bulk under the protection of PVP, which favors the formation of well-dispersed NPs.

Effect of Ag-EHCB concentration. Figure 3 shows a group of UV-vis absorption spectra for the colloidal solutions obtained for Ag-EHCB complex concentrations from $2.32 \times 10^{-3}$ (Exp $10)$ to $2.32 \times 10^{-2} \mathrm{~mol} / \mathrm{L}(\operatorname{Exp} 12)$ at the same hydrazine concentrations. Once the concentration of Ag-EHCB reached at least $2.32 \times 10^{-3} \mathrm{~mol} / \mathrm{L}$, the maximum UV absorption spectra of the resulting plasmon peak of the silver NPs was seen at $420 \mathrm{~nm}$. At room temperature apparent changes in absorbance were detected during the first 20 min of the reaction with hydrazine. Increasing the concentration of the Ag-EHCB complex, the band shows a red shift in the maximum of the plasmon peak. For relatively high concentrations of Ag-EHCB $\left(2.32 \times 10^{-2}\right.$
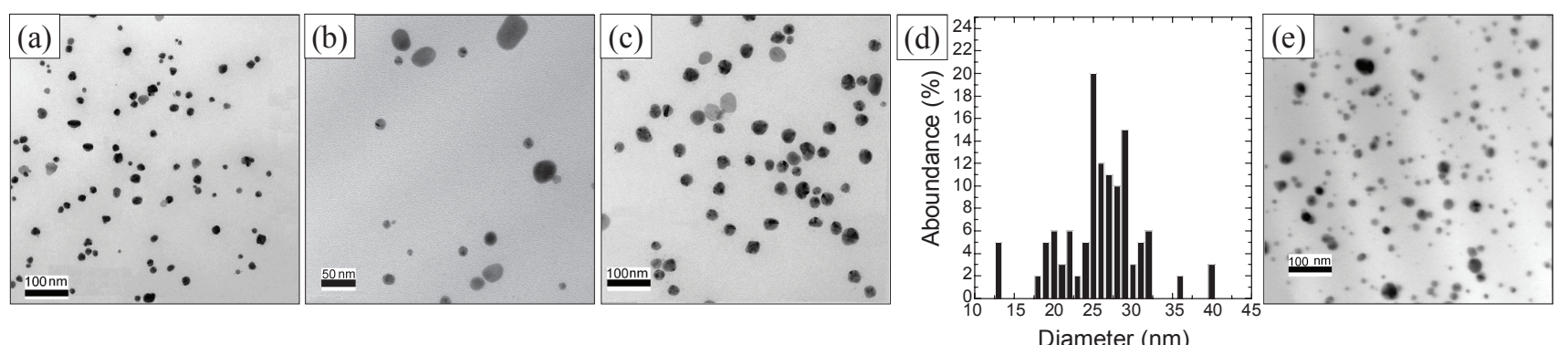

Figure 2. TEM image of a silver nanoparticle obtained from the Ag-EHCB complex $\left(4.64 \times 10^{-3} \mathrm{~mol} / \mathrm{L}\right)$ reduced with various amounts of hydrazine using PVP $\left(9.0 \times 10^{-3} \mathrm{~mol} / \mathrm{L}\right)$ : (a) Exp 2, (b) Exp 6, (c) Exp 4, (d) its histogram, and (e) Exp 5. 


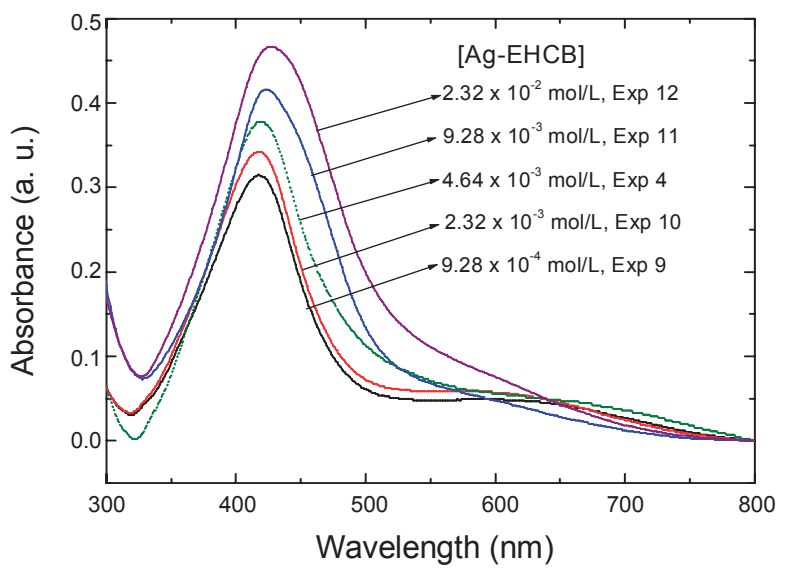

Figure 3. UV-vis absorption spectra of silver colloidal solutions obtained from various amounts of Ag-EHCB.
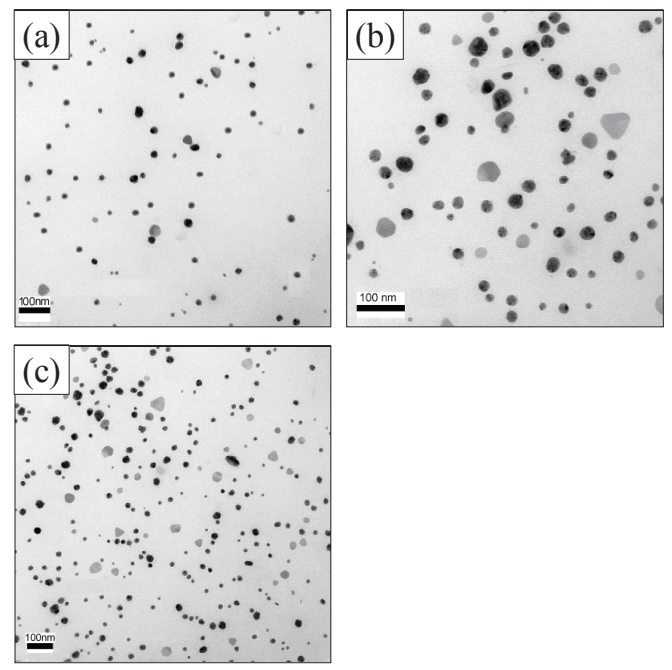

Figure 4. TEM image of silver colloidal solutions obtained from (a) $2.32 \times 10^{-3}$, (b) $9.28 \times 10^{-3}$, and (c) $2.32 \times 10^{-2} \mathrm{~mol} / \mathrm{L}$ of Ag-EHCB reduced with hydrazine $\left(1.0 \times 10^{-1} \mathrm{~mol} / \mathrm{L}\right)$ in the presence of PVP $\left(9.0 \times 10^{-3} \mathrm{~mol} / \mathrm{L}\right)$.

$\mathrm{mol} / \mathrm{L}$ ), a very broad absorbance band was observed, as can be seen in Figure 3. These features are associated with the size and distribution of the synthesized silver NPs. At lower Ag-EHCB concentrations, the absorption band narrowed and shifted below $420 \mathrm{~nm}$ (Exp 9).

The effect of Ag-EHCB quantity on the average colloidal size is shown in Figure 4. These data were obtained during the first $20 \mathrm{~min}$ of reaction time. Clearly there existed an optimal condition for obtaining smaller particle sizes at [hydrazine]/ [Ag-EHCB] ratios between 1.8 and 63. A more concentrated $\mathrm{Ag}$-EHCB complex solution was detrimental to the stability of these silver nanocolloids: the silver nanocolloid would grow from 5 - 30 (Figure 4(a)) to 50 - $80 \mathrm{~nm}$ (Figure 4(b)). A variety of shapes (e.g., spheres, triangles, hexagons, and polygons) with lengths of $30-80 \mathrm{~nm}$ and even $100 \mathrm{~nm}$ have been observed in such colloids. The results show that when the ratio of [hydrazine $] /[\mathrm{Ag}-\mathrm{EHCB}]$ was 10.78 , the NPs would eventually grew to a size of 20 - $50 \mathrm{~nm}$ even in the presence of PVP (Figure 4(c));

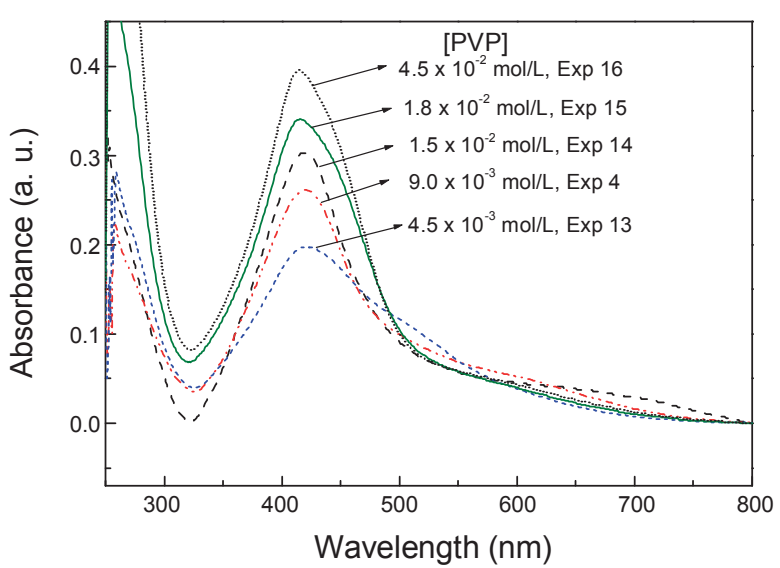

Figure 5. UV-vis absorption spectra of silver colloidal solutions obtained from Ag-EHCB in the presence of various amounts of PVP.
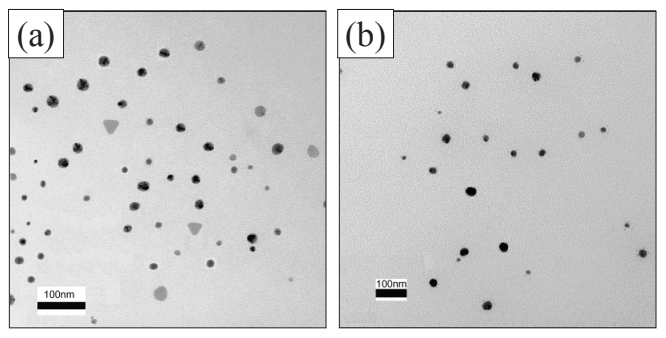

Figure 6. TEM image of silver colloidal solutions obtained from the Ag-EHCB complex $\left(4.64 \times 10^{-3} \mathrm{~mol} / \mathrm{L}\right)$ reduced with hydrazine $(1.0$ $\times 10^{-1} \mathrm{~mole} / \mathrm{L}$ ) in the presence of (a) $9.0 \times 10^{-3}$, and (b) $1.80 \times 10^{-2}$ $\mathrm{mol} / \mathrm{L}$ of PVP.

whereas if the ratio was larger than 10.78 , the particle size grew to $80 \mathrm{~nm}$ (Exp 11 and 12). This data set suggests that the amount of Ag-EHCB also affects the stability of the silver nanocolloids.

Effect of stabilizer PVP concentration. Figure 5 shows a group of UV-vis absorption spectra for the room temperature colloidal solution synthesis with different [PVP]/[Ag-EHCB] molar ratios using the same reduction time. Using a relatively high $[\mathrm{PVP}] /[\mathrm{Ag}-\mathrm{EHCB}]$ molar ratio a similar absorption band is observed, as shown in Figure 5. When the amount of stabilizer is insufficient, it cannot form a complete protection layer; thus, the particles will agglomerate easily to form large silver particles. In this case, the silver colloids would quickly coagulate into larger particles, probably due to the lower quantity of free PVP molecules. When more PVP disperser is used, it can quickly form a more perfect layer, which protects the particles from agglomeration and growth. When the ratio of [PVP]/[Ag-EHCB] decreases to 0.98 , a broad absorption band appears, centered near $420 \mathrm{~nm}$. Upon increasing the ratio from 0.98 to 9.8 , this band becomes more symmetrical, and a slight blue shift is observed in the maximum of the plasmon peak. These features are associated with the small size and uniform size distribution of the synthesized silver NPs.

As shown in Figure 6, the silver colloid obtained using a PVP concentration of $3.60 \times 10^{-2} \mathrm{~mol} / \mathrm{L}$ and a Ag-EHCB concentration of $4.64 \times 10^{-3} \mathrm{~mol} / \mathrm{L}$ consists of small NPs with spherical and ellipsoidal shapes at very wide size distributions (from 10 


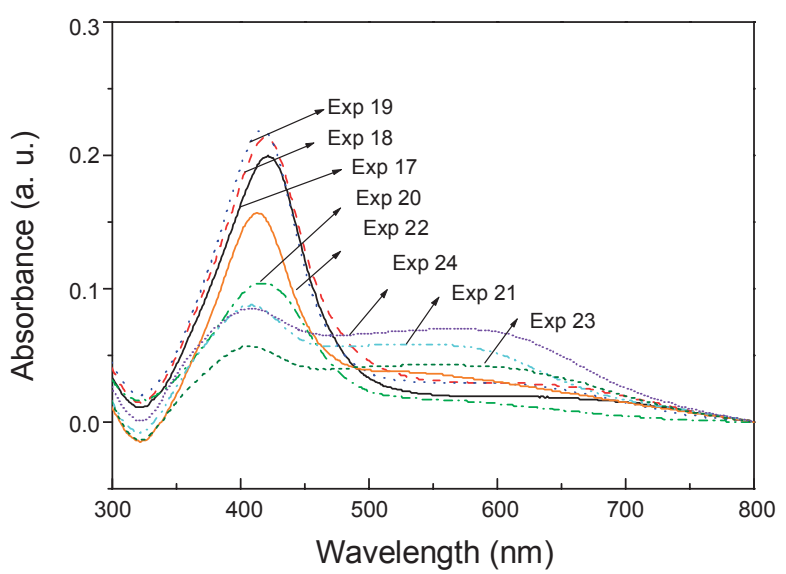

Figure 7. UV-vis absorption spectra of silver colloidal solutions obtained according to the various dropping times.

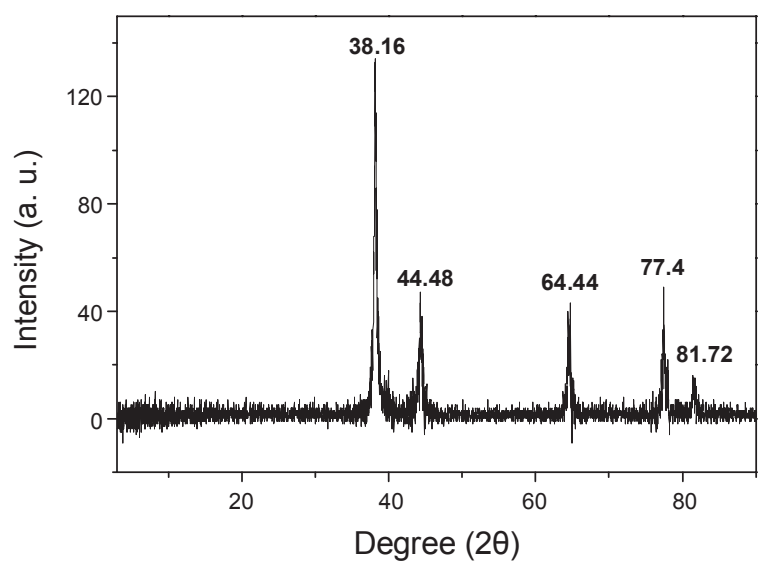

Figure 8. Powder X-ray diffraction pattern of a silver nanoparticle obtained from a silver nanocolloid solution (Exp 12).

to $20 \mathrm{~nm}$ ), as shown in Figure 6(a). A two-fold increase in silver concentration results in the formation of particles of different shapes. The average size of the spheres is $30 \mathrm{~nm}$, as shown in Figure 6(b).

Effect of reaction time. To monitor the formation of various size and shape silver NPs, we measured the absorption spectra of the colloid at different reduction reaction times. Figure 7 shows the absorption spectra of the silver NPs measured during the full reaction process at different times. There was an obvious change in the shape, position, and symmetry of the absorption peak according to the reduction time. The absorption band at longer dropping times of the hydrazine solution narrowed and continuously shifted to a shorter wavelength.

$\mathrm{X}$-ray diffraction and characterization. Due to its small size, the silver nanocolloid is quite difficult to separate from the mother solution by ordinary centrifuging techniques. However, we found that these colloids can be separated easily from the solution through centrifugation and filtration after the solution is mixed with 2-butanone. Presumably due to the poor solubility of PVP in 2-butanone, the PVP-protected silver colloids would agglomerate under these conditions, making the separation easier. If the precipitate was kept wet after separation, it could then be re-dispersed in de-ionized water. The XRD patterns of the powdered samples are shown in Figure 8. The XRD pattern shows five peaks positioned at $2 \theta$ values of $38.12^{\circ}, 44.48^{\circ}$, $64.44^{\circ}, 77.40^{\circ}$, and $81.72^{\circ}$. They are indexed to the (111), (200), (220), (311), and (222) planes. The XRD pattern matched with the literature values of pure silver.

The reduction process. Several routes have been proposed for the oxidation of hydrazine, which normally involve the evolution of nitrogen. ${ }^{40}$ Since we observed some gas evolution for the reaction at room temperature, we propose that the following mechanism takes place during the process. This mechanism is supported by a decrease of the basicity as the reaction proceeds, which indicates that the silver carbamate complex is progressively reduced. Actually, the carbamic acid formed can be converted easily to alkylammonium carbamate as

$$
\begin{array}{rl}
2 \mathrm{Ag}_{2}(\mathrm{OCONHR})_{2} \cdot & 4 \mathrm{NH}_{2} \mathrm{R}+\mathrm{H}_{2} \mathrm{NNH}_{2} \rightarrow \\
4 & \mathrm{Ag}+4 \mathrm{CO}_{2}+8 \mathrm{RNH}_{2}+\mathrm{N}_{2} \\
\mathrm{CO}_{2}+\text { excess } \mathrm{RNH}_{2} & \rightarrow \mathrm{RNHCOOH}+\mathrm{RNHCOO}^{-+} \mathrm{NH}_{3} \mathrm{R}
\end{array}
$$

Although this reaction is favored only at low temperature while at high temperature the reverse reaction is preferred. ${ }^{1} \mathrm{H}$ NMR measurements on the final particle free reaction product after reduction of $5.0 \times 10^{-3} \mathrm{M}$ silver carbamate complex at $100^{\circ} \mathrm{C}$, yielded the signals characteristic of alkylamine. At this high temperature, decomposition of carbamic acid and formation of 2-ethylhexylamine are expected. This reaction did not render an inorganic byproduct after reduction of silver carbamate complex, as shown in the chemical equation.

\section{Conclusion}

Colloidal silver particles in the nanometer size range were prepared in 2-propanol by reducing the Ag-EHCB complex with hydrazine in the presence of PVP stabilizer. Using the silver alkylcarbamate complex solution silver/PVP system allows very stable silver nanocolloids to be obtained in high concentrations in 2-propanol and in large scale, which is difficult to accomplish when using the other methods described in the literature. A facile and simple method for the preparation of a silver nanocolloid dispersion containing no inorganic ions was developed using silver carbamate complex.

\section{References}

1. Lee, P. C.; Miesel, D. J. Phys. Chem. 1982, 86, 3391.

2. Creigton, J.; Blatchford, C.; Albrecht, M. J. Chem. Soc. Faraday Trans. 1979, 75, 790.

3. Shirtcliffe, N.; Nickel, U.; Schneider, S. J. Colloid Interface Sci. 1999, 211, 122.

4. Toshima, N.; Harada, M.; Yonezawa, T.; Kushihashi, K.; Asakura, K. J. Phys. Chem. 1991, 95, 7448.

5. Pastoriza-Santos, I.; Liz-Marzan, L. M. Langmuir 1999, 15, $948-$ 951.

6. Rodriguez-Gattorno, G.; Diaz, D.; Rendon, L.; Hernandez-Segura, G. O. J. Phys. Chem. B 2002, 106, 2482.

7. Vasan, H. N.; Rao, C. N. R. J. Mater. Chem. 1995, 5, 1755.

8. Ayyappan, S.; Srinivasan Gopalan, R.; Subbana, G. N.; Rao, C. N. R. J. Mater. Res. 1997, 12, 398. 
9. Steiner, E. C.; Gilbert, J. M. J. Am. Chem. Soc. 1965, 87, 382.

10. Hong, H. K.; Park, C. K.; Gong, M. S. Bull. Korean Chem. Soc. 2010, 31, 1252.

11. Nie, S.; Emory, S. R. Science 1997, 275, 1102.

12. Nickel, U.; Castell, A.; Pöppl, K.; Shirtcliffe, N. Langmuir 2000, 16, 9087

13. Evanoff, D. D.; Chumanov, G., Jr. J. Phys. Chem. B 2004, 108, 13948.

14. Van Hyning, D. L.; Zukoski, C. F. Langmuir 1998, 14, 7034.

15. Cavicchioli, M.; Varanda, L. C.; Massabni, A. C.; Melnikov, P. Mater. Lett. 2005, 59, 3585.

16. Yang, N.; Aoki, K. Electrochim. Acta 2005, 50, 4868.

17. Kashiwagi, Y.; Yamamoto, M.; Nakamoto, M. J. Colloid Interface Sci. 2006, 300, 169.

18. Abe, K.; Hanada, T.; Yoshida, Y.; Tanigaki, N.; Takiguchi, H.; Nagasawa, H.; Nakamoto, M.; Yamaguchi, T.; Yase, K. Thin Solid Film 1998, 327, 524.

19. Yamamoto, M.; Nakamoto, M. J. Mater. Chem. 2003, 13, 2064.

20. Kashiwagi, Y.; Yamamoto, M.; Nakamoto, M. J. Colloid Interface Sci. 2006, 300, 169.

21. Szymańska, I.; Piszczek, P.; Szczęsny, R.; Szłyk, E. Polyhedron 2007, 26, 2440.

22. Liu, X.; Luc, S.; Zhang, J.; Cao, W. Thermochimica Acta 2006, $440,1$.

23. Monti, O. L. A.; Fourkas, J. T.; Nesbitt, D. J. J. Phys. Chem. B 2004, 108, 1604.

24. Singh, N.; Khanna, P. K. Mater. Chem. Phys. 2007, 104, 367.

25. Park, M. S.; Lim, T. H.; Jeon, Y. M.; Kim, J. G.; Joo, S. W.; Gong, M. S. Macromol. Res. 2008, 16, 308.
26. Park, M. S.; Lim, T. H.; Jeon, Y. M.; Kim, J. G.; Joo, S. W.; Gong, M. S. Sens. Actuators B 2008, 133, 166.

27. Hong, H. K.; Gong, M. S.; Park, C. K. Bull. Korean Chem. Soc. 2009, 30, 2669.

28. Park, H. S.; Park, H. S.; Gong, M. S. Polymer(Korea) 2010, 34 , 144.

29. Park, H. S.; Park, H. S.; Gong, M. S. Macromol. Res. 2010, $18,897$.

30. Grodzicki, A.; Łakomska, I.; Piszczek, P.; Szymánska, I.; Szłyk, E. Coord. Chem. Rev. 2005, 249, 2232.

31. Park, M. S.; Lim, T. H.; Jeon, Y. M.; Kim, J. G.; Joo, S. W.; Gong, M. S. J. Colloid Interface Sci. 2008, 321, 60.

32. Jeon, Y. M.; Cho, H. N.; Gong, M. S. Macromol. Res. 2009, 17, 2.

33. Lim, T. H.; Jeon, Y. M.; Kim, Gong, M. S. Polymer (Korea) 2009 , $33,33$.

34. Loginova, T. P.; Bronstein, L. M.; Mirzoeva, E. S.; Ezernitskaya, M. G.; Lokshin, B. V.; Genin, Y. V.; Valetsky, P. M. Polym. Sci. $1993,35,21$.

35. Hirai, H.; Chawanya, H.; Toshima, N. Reactive Polym. 1985, 3, 127.

36. Fendler, J. H. Nanoparticles and Nanostructured Films. Preparation, Characterization and Applications; Wiley: Weinheim, VCH, 1998.

37. Hirai, H.; Nakao, Y.; Toshima, N. J. Macromol. Sci. Chem. 1979, A13, 727 .

38. Mayer, A. B. R.; Mark, J. E. J. Polym. Sci. A: Polym. Chem. 1998, $35,197$.

39. Mayer, A. B. R. Mater. Sci. Eng, C: Biomimetic Mater., Sensors and Systems 1998, 6, 155.

40. Maillard, M.; Giorgio, S.; Pileni, M. P. Adv. Mater. 2002, 14, 1084. 